\title{
Recombinant neutralizing secretory IgA antibodies for preventing mucosal carriage and transmission of SARS-CoV-2
}

\section{Kathrin Göritzer ( $\sim$ kgoritze@sgul.ac.uk)}

Hotung Molecular Immunology Unit, 2Institute for Infection and Immunity, St. George's University of London, United Kingdom https://orcid.org/0000-0003-3742-028X

\section{Elisabetta Groppelli}

St. George's University of London

\section{Clemens Grünwald-Gruber}

Core Facility Mass Spectrometry, University of Natural Resources and Life Sciences, Vienna, Austria

\section{Rudolf Figl}

Core Facility Mass Spectrometry, University of Natural Resources and Life Sciences, Vienna, Austria

\section{Fengfeng Ni}

State Key Laboratory of Virology, Wuhan Institute of Virology, Center for Biosafety Mega-Science, Chinese Academy of Sciences, Wuhan, China

\section{Huimin $\mathrm{Hu}$}

State Key Laboratory of Virology, Wuhan Institute of Virology, Center for Biosafety Mega-Science, Chinese Academy of Sciences, Wuhan, China

\section{Yuncheng Li}

State Key Laboratory of Virology, Wuhan Institute of Virology, Center for Biosafety Mega-Science, Chinese Academy of Sciences, Wuhan, China

\section{Yalan Liu}

State Key Laboratory of Virology, Wuhan Institute of Virology, Center for Biosafety Mega-Science, Chinese Academy of Sciences, Wuhan, China

\section{Qinxue Hu}

State Key Laboratory of Virology, Wuhan Institute of Virology, Center for Biosafety Mega-Science, Chinese Academy of Sciences, Wuhan, China

\section{Rama Devudu Puligedda}

Lankenau Institute for Medical Research, Wynnewood, PA, United States

\section{Richard Strasser}

Department of Applied Genetics and Cell Biology, University of Natural Resources and Life Sciences, Vienna, Austria

\section{Scott Dessain}

Lankenau Institute for Medical Research, Wynnewood, PA, United States 


\section{Julian Ma}

Hotung Molecular Immunology Unit, Institute for Infection and Immunity, St. George's University of London, United Kingdom https://orcid.org/0000-0003-0767-0042

\section{Article}

Keywords: SARS-CoV-2, monoclonal antibodies, virus neutralization, mucosal antibodies, secretory IgA, passive immunisation, plantibodies, molecular pharming

Posted Date: November 29th, 2021

DOI: https://doi.org/10.21203/rs.3.rs-1053315/v1

License: (c) (i) This work is licensed under a Creative Commons Attribution 4.0 International License.

Read Full License 


\section{Recombinant neutralizing secretory IgA antibodies for preventing}

\section{2 mucosal carriage and transmission of SARS-CoV-2}

3 Kathrin Göritzer ${ }^{1 *}$, Elisabetta Groppelli ${ }^{2}$, Clemens Grünwald-Gruber ${ }^{3}$, Rudolf Figl $^{3}$, Fengfeng

$4 \mathrm{Ni}^{4}$, Huimin $\mathrm{Hu}^{4}$, Yuncheng $\mathrm{Li}^{4}$, Yalan $\mathrm{Liu}^{4}$, Qinxue $\mathrm{Hu}^{2,4}$, Rama Devudu Puligedda ${ }^{5}$, Richard

5 Strasser $^{6}$, Scott Dessain ${ }^{5}$ and Julian $\mathrm{Ma}^{1}$.

$6 \quad{ }^{1}$ Hotung Molecular Immunology Unit, ${ }^{2}$ Institute for Infection and Immunity, St. George's

$7 \quad$ University of London, United Kingdom

$8{ }^{3}$ Core Facility Mass Spectrometry, University of Natural Resources and Life Sciences, Vienna,

9 Austria

$10{ }^{4}$ State Key Laboratory of Virology, Wuhan Institute of Virology, Center for Biosafety Mega-

11 Science, Chinese Academy of Sciences, Wuhan, China

$12 \quad{ }^{5}$ Lankenau Institute for Medical Research, Wynnewood, PA, United States

$13{ }^{6}$ Department of Applied Genetics and Cell Biology, University of Natural Resources and Life

14 Sciences, Vienna, Austria

15

16

17 antibodies, secretory IgA, passive immunisation, plantibodies, molecular pharming; 


\section{Summary}

27 Passive delivery of antibodies to mucosal sites might be a valuable adjunct to COVID-19

28 vaccination to prevent infection, treat viral carriage, or block transmission. However,

29 monoclonal IgG antibody therapies, currently used for treatment of severe infections, are

30 unlikely to prove useful in mucosal sites where SARS-CoV-2 resides and replicates in early

31 infection. Here, we investigated the feasibility of producing neutralising monoclonal $\operatorname{IgA}$

32 antibodies against SARS-COV-2. We identified two class-switched mAbs that express well as

33 monomeric and secretory IgA variants with retained antigen binding affinities and increased

34 stability in mucosal secretions compared to their IgG counterparts. SIgAs had stronger virus

35 neutralisation activities than IgG mAbs and were able to reduce SARS-CoV-2 infection in an

36 in vivo murine model. Our findings provide a persuasive case for developing recombinant

37 SIgAs for mucosal application as a new tool in the fight against COVID-19. 
Introduction

COVID-19 is a mucosal infection caused by SARS-CoV-2. The virus replicates in the respiratory tract and is transmitted through respiratory droplets produced when an infected person coughs, sneezes, or talks. The most prominent symptoms of COVID-19 affect the respiratory system (continuous cough, shortness of breath), but in some cases sensory tissues in the upper respiratory tract are involved causing anosmia and loss of taste ${ }^{1}$. Additionally, gastro-intestinal symptoms (nausea, vomiting and diarrhea) are reported in $6 \%$ of adults and up to $20 \%$ in children ${ }^{2}$. Virus can be detected at all these sites as well as in urine ${ }^{3}$.

Infection with SARS-CoV-2 elicits systemic and mucosal immune responses ${ }^{4}$. Whilst attention has been focused on serum antibody responses which are dominated by $\mathrm{IgG}$, at mucosal sites such as the respiratory, gastrointestinal, and genitourinary tracts, immunoglobulin $\mathrm{A}(\operatorname{IgA})$ in the external secretions that bathe mucosal surfaces is the predominant antibody class ${ }^{5}$. Mucosal IgA in SARS-CoV-2 can be neutralising and long-lasting 4.

51 Various co-morbidities have been associated with diminished immune responses to SARSCoV-2, including immunosuppressive drugs to prevent transplant failure and diabetes ${ }^{6}$. Seroconversion following COVID-19 vaccination can also be compromised in these and similar patients ${ }^{7,8}$. In such circumstances, passive delivery of antibodies might be a valuable adjunct to COVID-19 vaccination, in which neutralising antibodies could be delivered directly to mucosal sites either to prevent infection, treat viral carriage or block transmission. Furthermore, topical delivery of antibodies could be useful to prevent carriage of virus in asymptomatic individuals.

59 Neutralising monoclonal IgG antibodies ${ }^{9}$ are already approved for systemic use in early SARS-

$60 \mathrm{CoV}-2$ treatment, but are unlikely to prove useful in mucosal fluids which are non-sterile and rich in endogenous and exogenous proteases ${ }^{10}$. For mucosal sites, $\operatorname{Ig} \mathrm{A}$ in the form of secretory 
$62 \operatorname{Ig} \mathrm{A}(\mathrm{SIg} \mathrm{A})$, would be preferred, as the polymeric complex is adapted for the harsh, unstable

63 external mucosal environment ${ }^{11}$. The potential for this approach has previously been

64 demonstrated in other model mucosal infections ${ }^{12,13,14}$.

65 However, monoclonal SIgA antibodies are technically challenging to produce. The first 66 recombinant approach to expressing secretory antibodies was in genetically modified plants ${ }^{15}$. 67 Other approaches have been described ${ }^{16,17,18}$, but still seem impractical or unaffordable for 68 commercial development. Some improvements to SIgA expression have been reported in 69 plants $19,20,21,22$ which still seems the most promising approach.

70 In this study, we investigated the feasibility of producing neutralising monoclonal IgA 71 antibodies against SARS-CoV-2. Starting with IgG class mAbs, we expressed monomeric and 72 secretory forms of $\operatorname{IgA}$ and compared these for their functionality and stability. Finally, we 73 assessed the potential use of SIgA to prevent SARS-CoV-2 infection in an in vivo model. 


\section{Experimental procedures}

\section{Construct design and cloning}

The plant codon-optimized sequences of the heavy and light chain variable regions of COVA215 (QKQ15273.1, QKQ15189.1), COVA1-22 (QKQ15169.1, QKQ15253.1), 2-15 (PDB: 7L57_H, 7L57_L) and 2E8 (manuscript submitted) IgG mAbs flanked with BsaI type II restriction sites were synthesized by GeneArt (Thermo Fisher Scientific, USA) ${ }^{23,24}$. Using Golden Gate assembly, the variable heavy chain sequences were cloned into pDONR based plasmids between a human Ig heavy chain leader sequence ('MELGLSWIFLLAILKGVQC') and either human gamma-1 (AAA02914.1), alpha-1 (AAT74070.1) or alpha-2m(1) (AAT74071.1) constant regions. Variable light chain fragments were inserted between the human light chain leader sequence ('MDMRVPAQLLGLLLLWLPGARC') and either human kappa constant regions for COVA2-15 variants (AAA58989.1) or lambda constants regions for COVA1-22, 2-15 and 2E8 variants (CAA40940.1) ${ }^{25}$. Full length heavy and light chain genes were separately subcloned into the binary high expression vector pEAQ-HT-DEST3 using Gateway cloning ${ }^{26}$. Human secretory component (SC) and joining chain (JC) constructs cloned separately into pEAQ-HT have been described previously ${ }^{14}$. The pEAQ-HT plant expression vectors containing the gamma and alpha heavy chains as well as the kappa and lambda light chains were transformed into Agrobacterium tumefaciens strain GV3101 (Leibniz Institut DSMZ-Deutsche Sammlung von Mikroorganismen und Zellkulturen GmbH, DSM 12364 ) by electroporation.

The construct for expression of the receptor binding domain (RBD) of the SARS-COV-2 spike (PDB-id: 6VYB, R319-F541) with a C-terminal 6xHis-tag cloned into pCAGGS mammalian expression vector was provided by Mark Dürkop from BOKU, Vienna. 
99 Agrobacteria containing the appropriate constructs were grown overnight at $28^{\circ} \mathrm{C}$ in Lysogeny-

100 Broth (LB) containing $25 \mu \mathrm{g} / \mathrm{mL}$ rifampicin and $50 \mu \mathrm{g} / \mathrm{mL}$ kanamycin. For expression of IgG

101 or monomeric IgA1 and IgA2 variants, the overnight cultures containing the respective 102 constructs for the heavy and light chain were diluted in infiltration buffer (10 mM MES, 10

$103 \mathrm{mM} \mathrm{MgSO}_{4}$, and $0.1 \mathrm{mM}$ acetosyringone) to an $\mathrm{OD}_{600}$ of 0.1 . For secretory $\operatorname{IgA}$ variants, heavy

104 and light chain constructs were diluted to an $\mathrm{OD}_{600}$ of 0.05 and mixed with the Joining chain

105 construct at an $\mathrm{OD}_{600}$ of 0.2 and the secretory component construct at an $\mathrm{OD}_{600}$ of 0.1 .

106 Agrobacteria solutions were then introduced into 6- to 8-week-old glycoengineered Nicotiana

107 benthamiana $\triangle \mathrm{XT} / \mathrm{FT}$ plants by vacuum infiltration ${ }^{27,28}$. Plants were grown in a controlled

108 environment room at $25^{\circ} \mathrm{C}$ with an 16/8-hour light/dark cycle. After 5 days, infiltrated leaf

109 material was harvested, and crude leaf extract was prepared by adding 3 volumes of ice-cold

110 phosphate-buffer saline (PBS) pH 7.4 containing $0.1 \%(\mathrm{v} / \mathrm{v})$ Tween in a blender. Homogenized

111 leaf material was passed through a Miracloth filter (Merck Millipore, Germany) and

112 centrifuged at $20000 \mathrm{x}$ g for $1 \mathrm{~h}$, followed by filtration through $0.45 \mu \mathrm{m}$ pore size filters

113 (Durapore membrane filter, Merck Millipore, Germany).

\section{Purification of $\operatorname{Ig} G$ and $\operatorname{IgA}$ variants from crude leaf extract}

115 Clarified leaf extracts were subjected to columns packed with either Pierce ${ }^{\mathrm{TM}}$ Protein A resin

116 for purification of $\operatorname{IgG}$ and COVA2-15 IgA variants or CaptureSelect ${ }^{\mathrm{TM}} \operatorname{IgA}$ affinity matrix

117 (both Thermo Fisher, US) for purification of 2E8 IgA variants equilibrated with PBS. Proteins

118 were eluted with $0.1 \mathrm{M}$ glycine $\mathrm{pH} 2.7$, followed by immediate addition of $10 \%(\mathrm{v} / \mathrm{v}) 1 \mathrm{M}$ Tris-

$119 \mathrm{HCl} \mathrm{pH} 9.0$ to neutralize the acidic $\mathrm{pH}$. Fractions containing the protein of interest were pooled

120 and dialyzed against PBS at $4{ }^{\circ} \mathrm{C}$ overnight using a dialyzing cassette with $10 \mathrm{kDa}$ molecular-

121 weight cut off (Slide-A-Lyzer, Thermo Scientific, US). Pooled and dialyzed protein fractions

122 were concentrated using Amicon centrifugal filters with a MWCO of $100 \mathrm{kDa}$ (Merck

123 Millipore, Germany) and subjected to size-exclusion chromatography (SEC) on a HiLoad 
124 16/600 Superdex 200 pg column (GE Healthcare, USA) equilibrated with PBS pH 7.4 connected to an ÄKTA pure (GE Healthcare, USA) FPLC system.

\section{Expression and purification of RBDHis}

127 For production of the recombinant receptor binding domain of the SARS-CoV-2 spike protein,

128 Expi293F ${ }^{\mathrm{TM}}$ cells were maintained and transfected according to the manufacturer's manual in

129 FreeStyle ${ }^{\mathrm{TM}}$ expression medium (all Thermo Fisher, US). High-quality plasmid preparations

130 were obtained using a Plasmid Midi kit (QUIAGEN, US). For the transfection of $200 \mathrm{~mL}$

131 culture with a cell density of $3.0 \times 10^{6}$ cells $/ \mathrm{ml}$, a total of $200 \mu \mathrm{g}$ plasmid DNA were mixed in 4

$132 \mathrm{~mL}$ OptiPro $^{\mathrm{TM}} \mathrm{SFM}$ medium and combined with another $4 \mathrm{~mL}$ OptiPro $^{\mathrm{TM}}$ containing $640 \mu \mathrm{L}$

133 ExpiFectamin (all Thermo Fisher, US). The mixture was incubated for 15 minutes before

134 gradual introduction to the cells. The culture was incubated for 7 days at $37^{\circ} \mathrm{C}$ in a humidified

135 atmosphere with $8 \% \mathrm{CO}_{2}$ on an orbital shaker rotating at $125 \mathrm{rpm}$. The supernatant containing

136 the secreted soluble protein was harvested by centrifugation at $20000 \mathrm{x} \mathrm{g}$ for 30 minutes at

$1374{ }^{\circ} \mathrm{C}$ and additionally filtrated through a 0.45 um Durapore membrane filter (Merx Millipore,

138 Germany). Clarified cell supernatant was diluted 1:2 in loading buffer (20 mM Tris, $500 \mathrm{mM}$

$139 \mathrm{NaCl}$ and $10 \mathrm{mM}$ imidazole). The solution was loaded onto a $5 \mathrm{~mL}$ HisTrap HP column (GE

140 Healthcare, US) equilibrated with 5 column volumes of loading buffer, and bound protein was

141 eluted by applying buffer containing $20 \mathrm{mM}$ Tris, $500 \mathrm{mM} \mathrm{NaCl}$ and $300 \mathrm{mM}$ imidazole.

142 Fractions containing the protein of interest were pooled and dialyzed against $\mathrm{PBS}$ at $4^{\circ} \mathrm{C}$

143 overnight using a dialyzing cassette with $10 \mathrm{kDa}$ molecular-weight cut off (Slide-A-Lyzer,

144 Thermo Scientific, United States). Pooled and dialyzed protein fractions were concentrated 145 using Amicon centrifugal filters with a MWCO of 100 kDa (Merck Millipore, Germany). 
$1485 \mu \mathrm{g}$ of purified mAbs were resolved on a NuPage 4-12\% Bis/Tris gel (Life Technologies,

149 UK) and stained with InstantBlue (Expedeon, UK).

150

151 For quantification of $\operatorname{IgG}$ and $\operatorname{IgA}$ mAbs in clarified crude extract of infiltrated $N$. benthamiana

152 plants ELISA plates were coated with $250 \mathrm{ng} /$ well anti-human gamma chain antiserum in PBS pH 7.4 at $4{ }^{\circ} \mathrm{C}$ overnight, respectively. After blocking with PBS containing $2 \%(\mathrm{w} / \mathrm{v})$ BSA and $0.1 \%$ Tween $20(\mathrm{v} / \mathrm{v})$ clarified crude plant extracts were added to the wells in normalized concentrations and incubated for $1.5 \mathrm{~h}$ at $37^{\circ} \mathrm{C}$. As standards, IgG1/kappa or IgG1/lambda isolated from human myeloma plasma (15154, I5029, Sigma, US), purified human IgA (P80-102, Bethyl, US) and IgA from human colostrum (I2363, Sigma, US) were used. Detection of secretory $\operatorname{IgA}$ variants was with mouse anti-secretory component $(\operatorname{Ig} \mathrm{A})$ antibody (SAB4200787, Sigma, US), followed by HRP-labeled anti-mouse antibody (SAB5300168, Sigma, US). For IgG and monomeric IgA variants, HRP-labeled anti-kappa (A18853, Invitrogen, US) or lambda light chain (ab200966, Abcam, UK) antisera were used.

163 After incubation for $1 \mathrm{~h}$ at $37^{\circ} \mathrm{C}$ plates were developed using TMB (Thermo Fisher, US) 164 substrate, the reaction was stopped with $2 \mathrm{M} \mathrm{H}_{2} \mathrm{SO}_{4}$ and read-out was an Infinite F200 Pro plate reader (Tecan, $\mathrm{CH})$ at $450 \mathrm{~nm}$ wavelength.

For determination of the ratio of functional and fully assembled SIgA to total IgA in each size-

167 exclusion fraction, similar ELISA assays were performed. Capture was with $150 \mathrm{ng} / \mathrm{well}$ 168 purified recombinant RBDHis or anti-alpha HC antibody (ab97211, Abcam, UK). Purified $169 \mathrm{mAbs}$ were diluted to $2 \mu \mathrm{g} / \mathrm{mL}$ in blocking solution and added to RBD and anti-IgA coated 170 plates in normalized concentrations, and incubated for $1.5 \mathrm{~h}$ at $37^{\circ} \mathrm{C}$. Detection of secretory component or antibody kappa or lambda chains was as described above. 
172 To determine the binding of the purified recombinant mAbs to SARS-CoV-2 RBD the ELISA

173 plates were coated with $150 \mathrm{ng} /$ well purified recombinant RBD-His and purified mAbs were

174 added to the wells in normalized concentration as above. For detection, HRP-labeled anti-

175 human kappa or lambda light chain antibody were used as above. Half-maximal concentration

$176\left(\mathrm{EC}_{50}\right)$ was calculated in GraphPad Prism 9.0.

\section{Competitive ELISA}

178 To determine the capability of purified mAbs to inhibit binding of RBD-His to the Ace2 179 receptor a competitive binding ELISA was performed. Purified Ace2-Fc was kindly provided 180 by Elisabeth Lobner (BOKU Vienna) and $500 \mathrm{ng} /$ well were coated on an ELISA plate at $4^{\circ} \mathrm{C}$ 181 overnight, followed by blocking with PBS containing 2\% (w/v) BSA and $0.1 \%$ Tween 20 (v/v).

182 RBD-His was incubated with varying molar ratios of the different mAbs starting with 2:1

183 [mAbs:RBD-His] reducing to $0.007: 1$ for $1 \mathrm{~h}$ at $37^{\circ} \mathrm{C}$ before addition to the wells. Binding of 184 RBDHis to Ace2-Fc was detected using an HRP-labeled anti-His antibody (71840, Sigma, US) 185 and plates were developed as described above.

Surface plasmon resonance (SPR) spectroscopy

187 The binding kinetics of plant-produced IgG and IgA mAbs to SARS-CoV-2 RBD-His were 188 determined on a BIAcore X-100 instrument (GE healthcare, Chalfont St Giles, UK) at $25{ }^{\circ} \mathrm{C}$ 189 with buffer HBS-EP+ (10 mm HEPES, pH 7.4, $150 \mathrm{mM} \mathrm{NaCl}, 3 \mathrm{mM}$ EDTA and 0.05\% 190 surfactant P-20). The monoclonal mouse anti-His antibody (SAB2702220, Sigma, US) was 191 immobilized onto a CM5 chip with standard amine coupling. Purified RBD-His was diluted in 192 HBS-EP+ buffer and injected at a concentration of $1 \mu \mathrm{g} / \mathrm{mL}$ for $30 \mathrm{sec}$ at the flow rate of $19330 \mu \mathrm{L} / \mathrm{min}$, followed by injection of 5 different concentration of each mAb with a flow rate of $19430 \mu \mathrm{L} / \mathrm{min}$. The second lowest concentration was repeated to ensure reproducibility, and 195 allowed to dissociate before regeneration with $10 \mathrm{mM}$ Glycine $\mathrm{pH} 1.7$ for $1 \mathrm{~min}$ at the flow rate 

of $10 \mu \mathrm{L} / \mathrm{min}$. Referenced and blanked sensorgrams were fitted with BIAcore Evaluation software using a 1:1 Langmuir model of binding. Each assay was performed in duplicate.

\section{Mass spectrometry}

199 A total of $20 \mu \mathrm{g}$ purified protein was reduced, S-alkylated and digested with trypsin (Promega, 200 USA). Glycopeptides were then analysed by capillary reversed-phase chromatography and 201 electron-spray mass spectrometry using an Agilent Series 6560 LC-IMS-QTOFMS instrument as reported previously ${ }^{29}$.

\section{MAb stability assays in human saliva}

204 Saliva was donated by two healthy volunteers and processed immediately after collection.

205 Neither donor had a previous natural infection with SARS-CoV-2 but both had received a two206 doses vaccination regime and their salivas had been shown to contained low levels of RBD 207 specific IgG but not SIgA antibodies (Ma, personal communication). The saliva was clarified by centrifugation at $3000 \mathrm{x}$ g for 15 minutes. Supernatants were collected and aliquoted into $100 \mu \mathrm{L}$ aliquots before being mixed with $10 \mu \mathrm{g}$ of each IgG and SIgA mAb variant in a volume

210 less than $5 \mu \mathrm{L}$. Following the immediate collection of a $15 \mu \mathrm{L}$ sample ( 0 minutes time-point), 211 antibody/saliva solutions were incubated at $37^{\circ} \mathrm{C}$ and sampled at 5, 60, 150, 240 and 1440

212 minutes. Samples were analyzed using a sandwich ELISA assay as described above, using 213 plates coated with $150 \mathrm{ng} /$ well purified recombinant RBD-His in PBS $\mathrm{pH}$ 7.4. The mAbs/saliva 214 solutions were diluted in blocking buffer 1:1000, added to the wells in normalized 215 concentrations and incubated for $1.5 \mathrm{~h}$ at $37^{\circ} \mathrm{C}$. The corresponding purified $\mathrm{mAb}$ in PBS buffer 216 with known concentration was used as control. IgG and SIgA mAbs were detected using HRP217 labelled mouse anti-IgG Fc (AP113P, Merck, Germany) and mouse anti-secretory component 218 (IgA) antibody (SAB4200787, Sigma, US), followed by HRP-labelled anti-mouse antibody 219 (SAB5300168, Sigma, US), respectively. 
221 Vero E6 cells stably expressing ACE-2 and TMPRSS-2 were obtained by NIBSC, UK and 222 grown in Dulbecco-MEM supplemented with $10 \%$ heat inactivated foetal calf serum (FCS, 223 Gibco, Thermo Fisher Scientific, US), penicillin (100 U/ml, Sigma), streptomycin $(100 \mu \mathrm{g} / \mathrm{ml}$, 224 Sigma), Hygromycin B $(250 \mu \mathrm{g} / \mathrm{ml}$, Thermo Fisher Scientific, US) and G418 $(250 \mu \mathrm{g} / \mathrm{ml}$, 225 Thermo Fisher). For plaque reduction assays, cells were seeded to obtain confluent monolayers $226\left(10^{5}\right.$ cells/well in 12 -well plates) and allowed to settle overnight. Monolayers were visually 227 inspected before use.

SARS-CoV-2 (England/2/2020) was obtained from Public Health England, UK and passaged in Vero E6 stably expressing ACE-2 and TMPRSS-2. Virus stocks were quantified with a standard plaque assay and expressed as plaque forming units per $\mathrm{ml}(\mathrm{pfu} / \mathrm{ml})$. Purified mAbs were serially diluted ten-fold starting at $15-20 \mu \mathrm{g} / \mathrm{ml}$ in Dulbecco-MEM with $2 \%$ FCS and incubated for $1 \mathrm{~h}$ at $37^{\circ} \mathrm{C}$ with $30-40$ plaque forming units (pfu) of SARS-CoV-

2 (England/2/2020). After incubation, the virus-antibody mixture was transferred onto a confluent monolayer of Vero-E6 cells expressing ACE-2 and TMPRSS-2 (NIBSC, UK). After 60 min adsorption at $37^{\circ} \mathrm{C}$, the inoculum was removed and replaced with an overlay containing growth medium (D-MEM with 10\% FCS) and 0.8\% Avicel (Sigma). The monolayers were incubated at $37{ }^{\circ} \mathrm{C}, 5 \% \mathrm{CO} 2$ for $48 \mathrm{~h}$ and then fixed and stained with paraformaldehyde $10 \%$ (Sigma) and crystal violet (1x, Sigma), respectively. Plaques were counted and expressed as \% of a neutralising positive control (WHO International Standard for anti-SARS-CoV-2

240 immunoglobulin, 20/136, NIBSC, UK). Percentage neutralisation (inhibition) was calculated in MS Excel and GraphPad Prism 9.0.

29 8-week-old male hACE2 transgenic mice (C57BL/6J) (T037630, GemPharmatech Co., 
mice were split into seven groups $(n=3-6)$ for prophylactic evaluation, as described in (Figure

246 5A). Mice without any challenge and treatment served as blank control (blank, $n=3$ ). Mice

247 challenged with SARS-CoV-2 but only PBS buffer as treatment were taken as infection control 248 (PBS, n=4). $250 \mu \mathrm{g}$ human serum IgG (I4506, Merck, Germany, IgG isotype control, $\mathrm{n}=4$ ) and 249 human colostrum IgA (I2636, Sigma, US, IgA isotype control, n=4) were administered 250 intranasally $24 \mathrm{~h}$ prior to infection and served as isotype treated controls. For the prophylactic group, IgG, SIgA1 or SIgA2 at a dose of $250 \mu \mathrm{g} / \mathrm{mouse}$ (average of $10 \mathrm{mg} / \mathrm{kg}$ ) was administered intranasally $24 \mathrm{~h}$ before infection (COVA2-15 IgG, SIgA1 and SIgA2, $-24 \mathrm{~h}, \mathrm{n}=4$ or 6). Body weight of each mouse was measured daily. The mice were sacrificed 6 days post infection (dpi) or at the humane endpoint. Lungs were collected for viral load determination and tissue sections for histopathology. Haematoxylin and eosin staining (H\&E) and immunohistochemical (IHC) staining were performed, respectively.

Viral load measurement by quantitative RT-PCR

Viral load was detected by quantitative real-time PCR (qRT-PCR) as described previously ${ }^{30}$. Briefly, lung homogenates were prepared by homogenizing perfused whole lung using an electric homogenizer. The supernatant was collected, and total RNA was extracted. Each RNA sample was reverse transcribed to cDNA with RT-PCR Prime Script Kit (Takara, Japan). The cDNA was used in a qRT-PCR reaction with the TaqMan Universal PCR Master Mix (Life Technologies, US), a TaqMan probe (5'-FAM- CAGGTGGAACCTCATCAGGAGATGC -MGB-3'), and primers designed to target the orflab gene of SARS-CoV-2 (5'GTGARATGGTCATGTGTGGCGG -3' and 5'- CARATGTTAAASACACTATTAGCATA -

$2663^{\prime}$ ). The samples were run in triplicate on an ABI 7900 Real-Time System (Applied 267 Biosystems, Thermo Fisher Scientific). The following cycling conditions were used: 1 cycle of $50{ }^{\circ} \mathrm{C}$ for $2 \mathrm{~min}, 1$ cycle of $95{ }^{\circ} \mathrm{C}$ for $10 \mathrm{~min}$, and 40 cycles of $95{ }^{\circ} \mathrm{C}$ for $15 \mathrm{sec}$ and $58{ }^{\circ} \mathrm{C}$ 269 for $1 \mathrm{~min}$. The virus titer was determined by comparison with a standard curve generated using 
270 RNA extracted from a serially diluted reference viral stock. All experiments were performed 271 in a Biosafety Level 3 facility.

272 Immunohistochemical staining of SARS-CoV-2-infected cells in tissues

273 Lung tissues were immersed in 10\% neutral buffered formalin (Z2902, Sigma, US) for $24 \mathrm{~h}$.

274 After the formalin fixation, the tissues were placed in $70 \%$ ethanol and subsequently embedded 275 with paraffin. Tissue sections (4- $\mu \mathrm{m}$ thick) were used for immunohistochemical staining for

276 SARS-CoV-2 detection using the Coronavirus nucleocapsid antibody (40143-MM05, Sino

277 Biological, China). Images were obtained by OLYMPUS IX73 using HCImage Live $(\times 64)$

278 software and analyzed by ImageJ (NIH).

279 Study approval

280 All animals infected by SARS-CoV-2 were handled in Biosafety Level 3 animal facilities in 281 accordance with the recommendations for care and use of the Institutional Review Board of 282 Wuhan Institute of Virology of the Chinese Academy of Sciences (Ethics Number: 283 WIVA11202003). All the authors declare their compliance with publishing ethics. 


\section{Recombinant production of anti-SARS-CoV-2 mucosal antibodies in plants}

286 We generated a panel of four anti-SARS-CoV-2 monomeric IgA and secretory IgA antibodies 287 targeting different epitopes on the spike protein. The variable regions of the well-characterized, neutralizing SARS-CoV-2 specific IgG antibodies COVA2-15 ${ }^{23}$, COVA1-22 ${ }^{23}, 2-15^{24}$ and 2E8 were cloned onto IgA1 and IgA2 constant domains for transient expression in glycoengineered $N$. benthamiana $\Delta \mathrm{XT} / \mathrm{FT}$ plants that are almost completely deficient in $\beta 1,2-$ xylosylation and core $\alpha 1,3$-fucosylation ${ }^{27}$. Light and heavy chain pairs were co-expressed in the presence and absence of the joining chain (JC) and secretory component (SC) to either obtain monomeric or secretory IgA. Immunoblot analysis and ELISA showed highest accumulation of recombinant protein after 5 to 6 days post infiltration. Expression levels of all monomeric $\operatorname{IgA} 1$ and $\operatorname{Ig} \mathrm{A} 2$ variants were high and approached those of their $\operatorname{IgG}$ counterparts, and all were functional in terms of binding to the SARS-CoV-2 spike protein (Figure 1A, Figure S1). However, assembly into multimeric secretory IgA when the JC and SC were coexpressed differed significantly and was highest for COVA2-15, followed by $2 \mathrm{E} 8$ and was reduced for COVA1-22 and 2-15 (Figure 1A). COVA2-15 and 2E8 variants were therefore selected for further analysis and characterization.

After affinity purification all IgG and IgA isotypes of COVA2-15 and 2E8 were subjected to size-exclusion chromatography. Both COVA2-15 and 2E8 IgG variants display single monodisperse peaks at the expected retention time for proteins with a mass of $\sim 150 \mathrm{kDa}$ (Figure 1B, dark grey shaded area). COVA2-15 and 2E8 monomeric IgA variants also display a major peak corresponding to the monomeric structural unit with additional minor peaks at lower retention times representing high molecular weight aggregates (Figure S2). Coinfiltration of IgA with the JC and SC result in a major peak with minor shoulders at earlier retention times (Figure 1B, green/blue shaded area) as well as a second peak representing non- 
assembled monomeric IgA (Figure 1B, light shaded area). Each of the eluted fraction was analyzed by ELISA to determine the ratio of fully functional and assembled secretory IgA.

311 Recombinant IgAs were captured with RBD and detected with anti-SC antibody and compared

312 to total IgA by using an anti-IgA heavy chain antibody for capture and an anti-kappa or lambda

313 light chain antibody for detection (Figure 1B, grey bars). Thereby it was shown that the major 314 peak and its shoulder at higher retention time (green/blue shaded area) of all variants contains 315 fully assembled and functional SIgA, while the peak shoulder observed for COVA2-15 SIgA1 and $\operatorname{SIgA} 2$ at an earlier retention time likely contains non-functional high molecular weight aggregates (HMWA). In general, formation of multimeric COVA2-15 and 2E8 IgA variants was very efficient compared to COVA1-22 and 2-15 (Figure S3) and previous reports of other multimeric IgA variants in plants ${ }^{19,21,22}$, whereas COVA2-15 SIgA1 and SIgA2 displayed better assembly than their 2E8 counterparts.

Size-exclusion chromatography fractions containing either the secretory and monomeric IgA species were pooled and were further analyzed using non-reducing SDS-PAGE (Figure 1C). Under non-reducing conditions both COVA2-15 and 2E8 mIgA1 and mIgA2 show a predominant band at a molecular mass around $160 \mathrm{kDa}$ representing the fully assembled monomer. Secretory IgA1 and IgA2 variants display a broad band at the expected size of 360 to $400 \mathrm{kDa}$. Monomeric IgA2 variants displayed additional bands at around $100 \mathrm{kDa}$ and 45 $\mathrm{kDa}$, which likely represent heavy and light chain dimers as the $\operatorname{IgA} 2 \mathrm{~m}(1)$ isotype used here does not have disulfide bridges linking the heavy and light chains, which are only associated through non-covalent intermolecular interactions ${ }^{29}$. The additional bands observed for monomeric $\operatorname{Ig} \mathrm{A} 2$ were not observed to the same extent for secretory $\operatorname{Ig} \mathrm{A} 2$. 
333 IgA is a heavily glycosylated protein with two predicted $N$-glycosylation sites in the $\operatorname{IgA} 1$

334 heavy chain, four in the $\operatorname{IgA} 2 \mathrm{~m}(1)$ heavy chain, one in the joining chain and six in the secretory

335 component. In addition, IgA1 has nine potential $O$-glycosylation sites in the proline-rich hinge

336 region, all of which are important post-translational modifications which confer many specific

337 properties of $\operatorname{IgA}$. To assess the glycosylation status of plant-produced $\operatorname{IgG}$, monomeric $\operatorname{Ig} \mathrm{A}$

338 and secretory IgA isotypes, the purified antibody variants were digested with trypsin and

339 subjected to LC-ESI-MS for analysis of site-specific $\mathrm{N}$-glycosylation and the presence of

340 modifications within the IgA1 hinge region (Figure 1D, Figure S4, Table S1). The single $N$ -

341 glycosylation site in the heavy-chain of COVA2-15 and 2E8 IgG was about 90\% occupied and

342 displays a very homogeneous glycosylation profile with the fully processed biantennary

343 complex-type GlcNAc2Man3GlcNAc2 $(\mathrm{GnGn})$ as major glycoform and lesser amounts of

344 GlcNAc1Man3GlcNAc2 (MGn/GnM).

345 All $N$-glycosylation sites in monomeric and secretory IgA1 and IgA2 heavy chains were fully

346 occupied except the C-terminal $N$-site present in the tailpiece of IgA (NVS) which was only 30

347 to $70 \%$ glycosylated as previously reported for plant-produced $\operatorname{IgA}{ }^{29}$. Besides bi-antennary

348 complex type structures (GnGn, MGn) the IgA heavy chains also contained high amounts of

349 oligomannosidic (Man5-9) and paucimannosidic (MM) structures as well as small amounts of

350 complex $N$-glycans carrying the plant-specific core $\alpha 1,3$-fucose resulting from the incomplete

351 silencing of $\alpha 1,3$-fucosyltransferase in the $N$. benthamiana $\Delta$ XT/FT line. Furthermore, some

352 site-specific processing can be observed for the NLT site in the CH2 domain of IgA1 and $\operatorname{IgA} 2$

353 which completely lacks $\alpha 1,3$-fucose and displays high amounts of oligomannosidic structures

354 which indicate insufficient secretion or inaccessibility for the respective glycosyltransferases,

355 which is even more pronounced when the secretory component is incorporated.

356 We were able to detect the single glycopeptide corresponding to the JC of the secretory $\operatorname{IgA}$

357 variants (Figure 1D, Table S2). The single $N$-glycan site in the JC of all variants was almost 
fully occupied and consisted of oligomannosidic structures, which differs from the very heterogeneously glycosylated JC of mammalian-produced SIgA containing complex-type

360 glycans with high levels of branching and incomplete sialylation ${ }^{22}$. The presence of 361 oligomannosidic $N$-glycans suggests incomplete processing of the JC $N$-glycans in the Golgi 362 of plants.

363 Furthermore, we were able to identify four individual tryptic glyco-peptides of the secretory component which were all fully occupied and displayed site-specific glycan processing (Figure 1D, Table S2). There was little difference between SC glycosylation of SIgA1 and SIgA2, or between COVA2-15 and 2E8 SIgAs. Site NGT exclusively contained oligomannosidic $\mathrm{N}$ glycans indicating reduced accessibility for processing at this site. Sites NDT, NYT and NVT mostly consisted of complex-type bi-antennary and paucimannosidic structures ( $\mathrm{MM}>\mathrm{GnGn}$, MGn), which are likely generated in a post-Golgi compartment by $\beta$-hexosaminidases, and completely lacked plant-specific $\alpha 1,3$-fucose ${ }^{31}$. On the hinge region of the plant produced monomeric and secretory $\operatorname{IgA} 1$ we detected the conversion of up to six proline residues to hydroxyproline and the addition of variable amounts of arabinoses in 30 to $50 \%$ of the converted hinge-regions (Figure S4, Table S3).

\section{Stability of anti-SARS-CoV-2 mAbs in human saliva}

376 Due to its unique structural features SIgA is expected to be better suited to survive and function on mucosal surfaces than $\operatorname{IgG}{ }^{32,33}$. To evaluate the stability of plant-produced anti-SARS-

$378 \mathrm{CoV}-2 \mathrm{IgG}$ and secretory $\operatorname{Ig} \mathrm{A}$ variants in human secretions, an in vitro experiment with COVA2-15 and 2E8 IgG, SIgA1 and SIgA2 was performed using saliva from two donors (Figure 2). Each mAb variant was incubated with saliva supernatant, incubated at $37^{\circ} \mathrm{C}$ and sampled at the times indicated. Time-point samples were analyzed for structural integrity and retained antigen binding capacity by sandwich ELISA capturing using RBD and detection with 
383 HRP-conjugated anti IgG-Fc or anti-secretory component. Although the rates of degradation

384 for both IgG and IgA variants based on COVA2-15 and 2E8 varied between experiments when

385 different saliva samples were used, intact IgG was lost at a consistently faster rate over the 386 experimental time-course than secretory IgA variants. The half-life of the SARS-CoV-2 IgG

387 mAbs were calculated using a one phase decay non-linear regression model. Half-lives of

388 COVA2-15 and 2E8 IgG variants were up to 30 minutes and were increased 5 to 10 -fold for COVA2-15 SIgAs and 2E8 SIgA2, but were difficult to determine for 2E8 SIgA1 as they did not decline to a plateau in the tested time-frame.

\section{Binding characteristics of different antibody formats to SARS-CoV-2 RBD}

393 Binding of the monomeric and secretory IgA formats to the SARS-CoV-2 receptor binding domain (RBD) was tested using ELISA and the half-maximal effective concentrations $\left(\mathrm{EC}_{50}\right)$ was determined (Figure 3A, Table S4). The binding behavior of monomeric and secretory $\operatorname{IgA} 1$ and $\operatorname{IgA} 2$ was comparable to their $\operatorname{IgG}$ counterpart, whereas COVA2-15 variants generally display stronger binding than 2E8 variants. In a competitive ELISA assay COVA215 and $2 \mathrm{E} 8 \mathrm{IgG}$ and $\operatorname{IgA} \mathrm{mAbs}$ were further analyzed for their capability to inhibit RBD binding to the ACE2-receptor (Figure 3B). Plant-produced IgG and IgA antibodies were able to inhibit RBD binding to ACE2-Fc using this assay, although 2E8 variants needed to be administered in higher molar ratios. Generally, secretory IgAs performed better, compared with monomeric $\operatorname{IgA}$ and $\operatorname{IgG}$ as expected due to their multivalency.

403 The binding kinetics of $\mathrm{IgG}$, monomeric and secretory $\operatorname{IgA}$ variants of COVA2-15 and 2E8 to 404 RBD were further investigated using surface plasmon resonance (SPR) spectroscopy. RBD was captured with a CM5 chip with immobilized anti-His antibody and different concentrations 406 of each mAb were injected in multi-cycle kinetic experiments and curves were fitted in a 1:1

407 binding model (Figure 3C). A rapid association $(k a)$ and very low dissociation rate $(k d)$ were 
characteristic for all COVA2-15 mAb variants, whereas a moderate association and faster 409 dissociation rate was observed for 2E8 IgG. Secretory IgA versions, particularly in the case of

$4102 \mathrm{E} 8$, displayed a more rapid association and a much-reduced dissociation rate with an up to 10-

411 fold increase in affinity $\left(K_{\mathrm{D}}\right)$ compared to $\operatorname{IgG}$ (Table 1). This avidity effect was not observed

412 so clearly for the COVA2-15 variants, likely due to the already near-irreversible nature of the

413 interaction of these monomeric formats with RBD.

414

Neutralization activity of different antibody formats

416 The neutralization ability of COVA2-15 and 2E8 IgG and IgA antibodies was investigated 417 using a live virus neutralization assay with a clinical isolate of SARS-COV-2 (England/2/2020) 418 propagated in Vero E6 cells stably expressing ACE2 and TMPRSS-2. Plaques were counted 419 and expressed as \% for non-neutralising control (Figure 4). All COVA2-15 mAb variants 420 showed high neutralization potential with 50\% inhibitory dose (ID 50 ) values ranging from 2 $\mathrm{ng} / \mathrm{mL}$ to $10 \mathrm{ng} / \mathrm{mL}$, which are in accordance to previously reported data of COVA2-15 IgG variants produced in a mammalian expression system ${ }^{23}$.

The RBD-targeting 2E8 mAbs showed reduced capability to block RBD binding to ACE2 in the competition ELISA (Figure 3B) suggesting a reduced virus neutralization potency. Indeed, the $\mathrm{IgG}$ version of $2 \mathrm{E} 8$ exhibited no inhibition at the tested concentrations, monomeric $\operatorname{IgA} 1$ and IgA2 were weak neutralizers (ID $50 \sim 1-2 \mu \mathrm{g} / \mathrm{mL}$ ) and only the secretory $\operatorname{IgA}$ variants (2E8 SIgA1 ID $50 \sim 5$ ng/mL, (2E8 SIgA2 ID $50 \sim 20 \mathrm{ng} / \mathrm{mL}$ ) had strong neutralizing activities (Figure 4B).

430 Efficacy of intranasally administered anti-SARS-CoV-2 mucosal antibodies in ACE2 mice

431 To compare the prophylactic efficacy of COVA2-15 IgG, SIgA1 and SIgA2 in vivo, mAbs 432 were administrated intranasally to hACE2 transgenic mice 24 hours prior to challenge with 
433 SARS-CoV-2 (Figure 5A). High levels of viral RNA $\left(3.4 \times 10^{6}\right.$ copies/mg) were detected in 434 the lungs of control and isotype treated control mice, which were significantly reduced in the 435 prophylactic groups, particularly those treated with $250 \mu \mathrm{g}$ (average of $10 \mathrm{mg} / \mathrm{kg}$ ) COVA2-15 436 IgG, as evidenced by real-time PCR (Figure 5B). Significant reduction in viral RNA was also 437 observed in mice treated with $250 \mu \mathrm{g}$ COVA2-25 SIgA1 or SIgA2. The results correlate with 438 clinical protection, with partial protection afforded by SIgA antibodies and full protection by 439 IgG (Figure 5C). Mice receiving COVA2-15 mAbs (IgG and SIgAs) treatment showed less 440 weight loss than the controls (Figure 5D). Histopathological analysis of lung tissues 441 demonstrated that SARS-CoV-2 induced lung lesions, focal infiltration of inflammatory cells 442 around bronchi and blood vessels (blue arrows) and alveolar septal thickening (green arrows) 443 in the control mice. There was also narrowing and collapse of the alveolar wall with creation 444 of larger cystic cavities. In the COVA2-15 IgG treated groups, there was little pathology but 445 the appearance of lungs in the SIgA treated groups resembled the PBS treated control group 446 more closely (Figure 5E). 


\section{Discussion}

449 In this study we investigated the feasibility of producing neutralizing mucosal IgA antibodies against SARS-CoV-2, performed a detailed biochemical and functional analysis of the recombinant antibodies, and explored their potential use to prevent infection in an in vivo model. We used the plant-based Nicotiana benthamiana $\Delta \mathrm{XT} / \mathrm{FT}$ expression platform for transient production of different monomeric and secretory IgA variants based on several different published IgG antibodies recognizing the SARS-CoV-2 spike protein. All of the monomeric IgG and IgA antibody variants expressed moderately to well and were functional in terms of antigen binding. However, the capacity for assembly into multimeric secretory $\operatorname{Ig} \mathrm{A}$ differed greatly between antibodies with different variable regions. COVA2-15 SIgAs displayed almost full assembly (80-90\%) and 2E8 variants demonstrated up to 70\% assembly into multimers, thereby exceeding yields and ratios of recombinantly produced SIgA to monomeric $\operatorname{IgA}$ in previous reports using plant- and mammalian-based expression ${ }^{22,34,35,36 .}$

461 SIgA antibodies based on 2-15 and COVA1-22 on the other hand showed very poor assembly

462 into multimers even though they only differ from COVA2-15 and 2E8 in the variable domain 463 sequences.

464 In previous studies it was shown that the JC incorporation is the limiting factor for secretory 465 IgA formation ${ }^{21,22,35}$. This is consistent with our finding, where we also did not observe an 466 increase of dimeric IgA when the amount of infiltrated JC was varied. Other factors that were 467 reported to contribute to dimer formation were the involvement of certain human chaperones 468 including ERp44 or MZB.1, certain structural features of the CH3 domains of IgA1 and $\operatorname{IgA} 2$ 469 and tailpiece glycosylation 21,22,37. Our data indicate that there are also additional factors 470 contributing to Joining chain incorporation and IgA dimerization which need to be investigated 471 further. 
472 Mucosal antibody variants COVA2-15 and 2E8 which displayed highest yield and assembly 473 capacity were selected for detailed characterization. Class-switching of COVA2-15 and 2E8 474 IgG to monomeric and secretory $\operatorname{IgA}$ variants did not significantly influence $\mathrm{EC}_{50}$ for binding 475 to the SARS-CoV-2 RBD despite higher valency of SIgAs. However, avidity effects were 476 apparent in SPR kinetic experiments in which the dissociation rate and consequently the 477 dissociation constant $\left(K_{\mathrm{D}}\right)$ of $2 \mathrm{E} 8 \mathrm{SIgA}$ compared to the IgG counterpart displaying a moderate 478 affinity, were much enhanced.

479 Strong binding to RBD and competition with the ACE2 receptor binding translated into potent 480 virus neutralization capacities of all COVA2-15 mAb variants, although no significant increase 481 from monomeric to multimeric antibody formats could be observed despite higher valency of antigen binding sites. This could be due to the already extremely high affinity of monomeric 483 Ig variants. The contrary was observed for 2E8 based antibodies, which showed moderate binding to RBD and reduced competition with ACE2 binding compared with COVA2-15. The monomeric Ig formats of $2 \mathrm{E} 8$ displayed little or moderate virus neutralisation, but the multimeric SIgA formats showed strong inhibition. The increased activity of monomeric IgA over IgG might result from the extended hinge region in $\operatorname{IgA} 1$ or other structural differences of the antibody classes. Furthermore, $\operatorname{IgA}$ and multimeric antibody formats could enhance inhibition of virus entry through other mechanisms such as steric hindrance or increased avidity $490 \quad{ }^{38}$, potentially offering a means of rescuing or re-purposing relatively poorly performing $\operatorname{IgG}$ $491 \quad$ antibodies $^{36}$.

492 Whilst increased valency might confer increased neutralization capacity for some antibody candidates, SIgA is also believed to have a longer half-life in mucosal secretions due to its 494 unique structural features making it less susceptible to proteolysis. SIgA also has unique 495 interactions with structural and functional components of the mucosa and displays non496 inflammatory properties ${ }^{32,33}$. Some of these characteristics are conferred by the extensive $N$ - 
497 glycosylation of heavy chains and secretory components of $\operatorname{IgA}{ }^{39}$, which in humans carries 498 mostly branched complex $N$-glycans with high levels of sialic acid and with seven putative 499 sites occupied in varying degrees ${ }^{40}$. This study confirmed that plants are capable of performing 500 these complex post-translational modifications with relatively high homogeneity compared to 501 mammalian production systems and mostly absent $N$-glycan modifications such as $\beta 1,2$ xylose and $\alpha 1,3$ fucose, which are commonly found in plants that have not been glycoengineered ${ }^{41}$, 42. The elongated hinge-region of plant-produced $\operatorname{IgA} 1$ on the other hand, exhibits plant504 specific conversion of prolines to hydroxyprolines followed by addition of unbranched 505 arabinose chains. Hydroxyproline residues are not found on human proteins such as $\operatorname{IgA} 1$ and concerns have been raised that the presence of arabinose chains may bear a risk of an unwanted immune response. Consistent with previous observations of increased half-life for IgA in the mucosa, plant-produced COVA2-15 and 2E8 SIgA variants were significantly more stable in saliva compared to their $\operatorname{IgG}$ counterparts ${ }^{19}$. Interestingly, SIgA1 and SIgA2 showed similar

510 rates of degradation, although in humans $\mathrm{SIgA} 1$ is more prone to degradation by proteases 511 produced by oral bacteria selectively cleaving sites in the extended hinge-region of $\operatorname{IgA} 1$, 512 leaving SIgA2 as the predominant isotype in mucosal secretions ${ }^{43,44}$. Here, the activity of 513 bacterial proteases on the extended IgA1 hinge-region might be reduced due to the conversion 514 of prolines to hydroxyprolines, which lie within the recognition sequence of many bacterial 515 proteases and are partially extended with arabinose chains, thereby potentially masking the 516 cleavage site ${ }^{45,46}$. To date there is only limited knowledge about safety and efficacy of the 517 repeated application of recombinant mAbs let alone plant-produced IgAs to mucosal surfaces 518 or influences of plant-specific modifications. However, repeated application of a plant-made 519 SIgA to the oral cavity did not cause any side effects ${ }^{12}$.

520 Neutralizing antibodies against SARS-CoV-2 are increasingly used in early treatment of severe 521 COVID-19, but only administered by the systemic rout. SIgAs applied topically to mucosal 
522 sites might provide a different, much earlier intervention to tackle viral carriage and 523 transmission. SARS-CoV-2 is mainly present in the nasopharynx and lungs ${ }^{47,48}$, so direct 524 administration to the upper respiratory tract might provide faster and more robust antiviral 525 activity in the sites, where the virus resides and replicates ${ }^{48,49}$. Here, the in vivo study addressed 526 protection against SARS-CoV-2 challenge in a hACE2 mouse model. This model has the 527 advantage of being strongly informative while being technically straight forward compared to 528 carriage and transmission blocking studies in other animal models. We demonstrated partial protection against SARS-CoV-2 infection in mice treated with plant-produced SIgA1 and

530 SIgA2, but also that the plant-produced IgG COVA2-15 provided complete protection in this 531 model. The apparent superiority of IgG might be due to limitations inherent with the study, 532 specifically the inability to directly compare antibody concentrations for IgG and SIgAs due to different ELISA formats used to quantify concentrations. Additionally, although the murine model is an invaluable system, the upper respiratory murine mucosal microbiota differs to that of humans and can result in an antibody class being favored over another.

536 In summary, we demonstrated that neutralising monoclonal IgA antibodies against SARS-

$537 \mathrm{CoV}-2$ can be produced as monomeric and secretory formats in a plant-based expression 538 system. We showed that these antibodies are able to maintain their structure and binding 539 affinities when incubated in the harsh environment of human saliva. Importantly, we showed 540 that these plant-generated antibodies have strong virus neutralisation activity and can reduce

541 SARS-CoV-2 infection in an in vivo murine model. Therefore, our preliminary data provide 542 strong evidence of the value of secretory $\operatorname{IgA}$ in clinical management and/or prevention of 543 COVID-19. 


\section{Declaration of interest}

546 The authors declare that the research was conducted in the absence of any commercial or

547 financial relationships that could be construed as a potential conflict of interest.

\section{$548 \quad$ Author contributions}

549 KG conducted most experiments, analysed the data, and wrote the draft manuscript. SD and

550 RP discovered and cloned 2E8 sequences. EG conducted the in vitro neutralization experiments. RS provided $N$. benthamiana $\Delta \mathrm{XF} / \mathrm{FT}$ plants and glycosylation expertise. $\mathrm{CG}, \mathrm{RF}$ performed site-specific glycan analysis. FN, HH, YL, YL and QH conducted the animal challenge experiments and evaluated the efficacy of mAbs in SARS-CoV-2 infected transgenic hACE2 mice. JM, KG designed the study, directed and financially supported the study, JM, $\mathrm{KG}, \mathrm{RS}$ and SD revised the manuscript. All authors critically reviewed the draft manuscript and approved the final version.

\section{Data availability statement}

Upon publication the variable domain gene sequences of the 2E8 antibody will be deposited in the Coronavirus Antibody Database, CoV-Ab-Dab (Oxford Protein Informatics Group) and

560 Genbank. Any additional information required to reanalyse the data reported in this paper is available from the lead contact upon request.

\section{Acknowledgements}

563 This work received generous support from the Sir Joseph Hotung Charitable Trust; the

564 European Union's Horizon 2020 programme under Grant Agreements 774078 (Pharma-

565 Factory) and 760331 (Newcotiana); and the Austrian Science Fund Erwin Schrödinger

566 Fellowship J-4583. The MS equipment was kindly provided by the EQ-BOKU VIBT GmbH

567 and the BOKU Core Facility mass spectrometry. 


\section{References}

600

601

602

603

604

605

606

607

608

609

610

1. Speth, M.M. et al. Olfactory Dysfunction and Sinonasal Symptomatology in COVID19: Prevalence, Severity, Timing, and Associated Characteristics. Otolaryngol Head Neck Surg, 194599820929185 (2020).

2. Jin, X. et al. Epidemiological, clinical and virological characteristics of 74 cases of coronavirus-infected disease 2019 (COVID-19) with gastrointestinal symptoms. Gut 69, 1002-1009 (2020).

3. Peng, L. et al. SARS-CoV-2 can be detected in urine, blood, anal swabs, and oropharyngeal swabs specimens. J Med Virol 92, 1676-1680 (2020).

4. Fröberg, J. et al. SARS-CoV-2 mucosal antibody development and persistence and their relation to viral load and COVID-19 symptoms. Nature communications 12 (2021).

5. Lamm, M.E. \& Phillips-Quagliata, J.M. Origin and homing of intestinal IgA antibodysecreting cells. J Exp Med 195, F5-8 (2002).

6. Suleyman, G. et al. Clinical Characteristics and Morbidity Associated With Coronavirus Disease 2019 in a Series of Patients in Metropolitan Detroit. JAMA Netw Open 3, e2012270 (2020).

7. Cucchiari, D. et al. Cellular and humoral response after MRNA-1273 SARS-CoV-2 vaccine in kidney transplant recipients. Am J Transplant 21, 2727-2739 (2021).

8. Grupper, A. et al. Humoral Response to the Pfizer BNT162b2 Vaccine in Patients Undergoing Maintenance Hemodialysis. Clin J Am Soc Nephrol 16, 1037-1042 (2021).

9. Weinreich, D.M. et al. REGN-COV2, a Neutralizing Antibody Cocktail, in Outpatients with Covid-19. New England Journal of Medicine 384, 238-251 (2020).

10. Woof, J.M. \& Kerr, M.A. The function of immunoglobulin A in immunity. The Journal of pathology 208, 270-282 (2006).

11. Mestecky, J. \& McGhee, J.R. Immunoglobulin A (IgA): molecular and cellular interactions involved in IgA biosynthesis and immune response. Adv Immunol 40, 153245 (1987).

12. Ma, J.K. et al. Characterization of a recombinant plant monoclonal secretory antibody and preventive immunotherapy in humans. Nat Med 4, 601-606 (1998). 
13. Berdoz, J., Blanc, C.T., Reinhardt, M., Kraehenbuhl, J.P. \& Corthesy, B. In vitro comparison of the antigen-binding and stability properties of the various molecular forms of IgA antibodies assembled and produced in CHO cells. Proc Natl Acad Sci U $S$ A 96, 3029-3034 (1999).

14. Teh, A.Y. et al. Investigation of a monoclonal antibody against enterotoxigenic Escherichia coli, expressed as secretory IgA1 and IgA2 in plants. Gut Microbes 13, 114 (2021).

15. Ma, J.K. et al. Generation and assembly of secretory antibodies in plants. Science (New York, N.Y.) 268, 716-719 (1995).

16. Bhaskara, V. et al. Efficient production of recombinant secretory IgA against Clostridium difficile toxins in CHO-K1 cells. Journal of Biotechnology 331, 1-13 (2021).

17. Puligedda, R.D. et al. Human IgA Monoclonal Antibodies That Neutralize Poliovirus, Produced by Hybridomas and Recombinant Expression. Antibodies (Basel) 9 (2020).

18. Boullier, S. et al. Secretory IgA-mediated neutralization of Shigella flexneri prevents intestinal tissue destruction by down-regulating inflammatory circuits. Journal of immunology (Baltimore, Md. : 1950) 183, 5879-5885 (2009).

19. Paul, M. et al. Characterization of a plant-produced recombinant human secretory IgA with broad neutralizing activity against HIV. MAbs 6, 1585-1597 (2014).

20. Virdi, V., Juarez, P., Boudolf, V. \& Depicker, A. Recombinant IgA production for mucosal passive immunization, advancing beyond the hurdles. Cellular and molecular life sciences : CMLS 73, 535-545 (2016).

21. Westerhof, L.B. et al. Transient Expression of Secretory IgA In Planta is Optimal Using a Multi-Gene Vector and may be Further Enhanced by Improving Joining Chain Incorporation. Front Plant Sci 6, 1200 (2015).

22. Göritzer, K. et al. Efficient N-Glycosylation of the Heavy Chain Tailpiece Promotes the Formation of Plant-Produced Dimeric IgA. Front Chem 8, 346 (2020).

23. Brouwer, P.J.M. et al. Potent neutralizing antibodies from COVID-19 patients define multiple targets of vulnerability. Science (New York, N.Y.) 369, 643-650 (2020).

24. Liu, L. et al. Potent neutralizing antibodies against multiple epitopes on SARS-CoV-2 spike. Nature 584, 450-456 (2020). 
662

663

664

665

666

667

668

669

670

671

672

673

674

675

676

677

678

679

680

681

682

683

684

685

686

687

688

689

690

691

692

693

694

695

696

25. Teh, A.Y.-H.C., L; Hu, Y; Kumru, O; Xiong, J; Bolick, DT; Joshi, SB; GrünwaldGruber, C; Altmann, F; Klempner, M; Guerrant, RA; Volkin, DB; Wang, Y; Ma, J KC. Investigation of a monoclonal antibody against enterotoxigenic Escherichia coli, expressed as secretory IgA1 and IgA2 in plants. Gut Microbes ISSN 1949-0976 in press (2020).

26. Peyret, H. \& Lomonossoff, G.P. The pEAQ vector series: the easy and quick way to produce recombinant proteins in plants. Plant molecular biology 83, 51-58 (2013).

27. Strasser, R. et al. Generation of glyco-engineered Nicotiana benthamiana for the production of monoclonal antibodies with a homogeneous human-like N-glycan structure. Plant biotechnology journal 6, 392-402 (2008).

28. Teh, A.Y., Maresch, D., Klein, K. \& Ma, J.K. Characterization of VRC01, a potent and broadly neutralizing anti-HIV mAb, produced in transiently and stably transformed tobacco. Plant biotechnology journal 12, 300-311 (2014).

29. Goritzer, K., Maresch, D., Altmann, F., Obinger, C. \& Strasser, R. Exploring sitespecific N-glycosylation of HEK293 and plant-produced human IgA isotypes. Journal of proteome research 16, 2560-2570 (2017).

30. $\mathrm{Wu}, \mathrm{X}$. et al. A potent bispecific nanobody protects hACE2 mice against SARS-CoV2 infection via intranasal administration. Cell reports, 109869 (2021).

31. Shin, Y.J. et al. Reduced paucimannosidic N-glycan formation by suppression of a specific beta-hexosaminidase from Nicotiana benthamiana. Plant biotechnology journal (2016).

32. Corthésy, B. Recombinant secretory immunoglobulin A in passive immunotherapy: linking immunology and biotechnology. Curr Pharm Biotechnol 4, 51-67 (2003).

33. Corthesy, B. Multi-faceted functions of secretory IgA at mucosal surfaces. Frontiers in immunology 4, 185 (2013).

34. Westerhof, L.B. et al. Monomeric IgA can be produced in planta as efficient as IgG, yet receives different N-glycans. Plant biotechnology journal 12, 1333-1342 (2014).

35. Lombana, T.N. et al. Production, characterization, and in vivo half-life extension of polymeric IgA molecules in mice. $m A b s$ 11, 1122-1138 (2019).

36. Sun, L. et al. Increased in vitro neutralizing activity of SARS-CoV-2 IgA1 dimers compared to monomers and IgG. Proceedings of the National Academy of Sciences 118, e2107148118 (2021). 
37. Xiong, E. et al. MZB1 promotes the secretion of J-chain-containing dimeric IgA and is critical for the suppression of gut inflammation. Proc Natl Acad Sci U S A 116, 1348013489 (2019).

38. Yang, H. \& Rao, Z. Structural biology of SARS-CoV-2 and implications for therapeutic development. Nature Reviews Microbiology (2021).

39. Brandtzaeg, P. Secretory IgA: Designed for Anti-Microbial Defense. Frontiers in immunology 4, 222 (2013).

40. Huang, J. et al. Site-specific glycosylation of secretory immunoglobulin A from human colostrum. Journal of proteome research 14, 1335-1349 (2015).

41. Stelter, S. et al. Engineering the interactions between a plant-produced HIV antibody and human Fc receptors. Plant biotechnology journal 18, 402-414 (2020).

42. Cabanes-Macheteau, M. et al. N-Glycosylation of a mouse IgG expressed in transgenic tobacco plants. Glycobiology 9, 365-372 (1999).

43. Breedveld, A. \& van Egmond, M. IgA and FcalphaRI: Pathological Roles and Therapeutic Opportunities. Frontiers in immunology 10, 553 (2019).

44. van Egmond, M. et al. IgA and the IgA Fc receptor. Trends in Immunology 22, 205$211(2001)$.

45. Novak, J. et al. Heterogeneity of O-glycosylation in the hinge region of human IgA1. Molecular immunology 37, 1047-1056 (2000).

46. Mócsai, R. et al. Prolyl Hydroxylase Paralogs in Nicotiana benthamiana Show High Similarity With Regard to Substrate Specificity. Frontiers in Plant Science 12 (2021).

47. Gallo, O., Locatello, L.G., Mazzoni, A., Novelli, L. \& Annunziato, F. The central role of the nasal microenvironment in the transmission, modulation, and clinical progression of SARS-CoV-2 infection. Mucosal Immunol 14, 305-316 (2021).

48. Higgins, T.S. et al. Intranasal Antiviral Drug Delivery and Coronavirus Disease 2019 (COVID-19): A State of the Art Review. Otolaryngol Head Neck Surg 163, 682-694 (2020).

49. Cunningham, S. et al. Nebulised ALX-0171 for respiratory syncytial virus lower respiratory tract infection in hospitalised children: a double-blind, randomised, placebo-controlled, phase 2b trial. Lancet Respir Med 9, 21-32 (2021). 
Figures

A

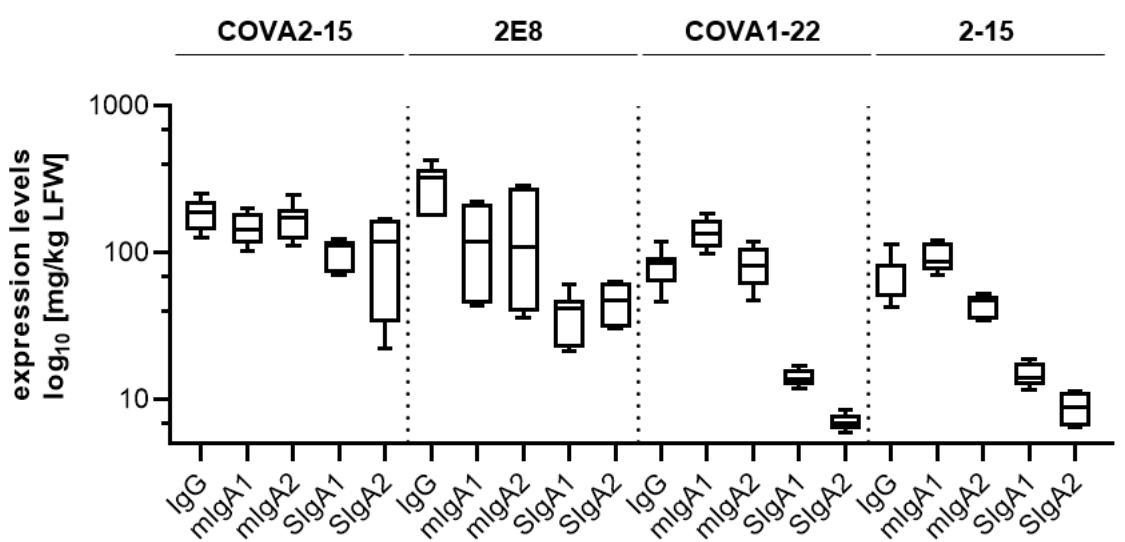

B

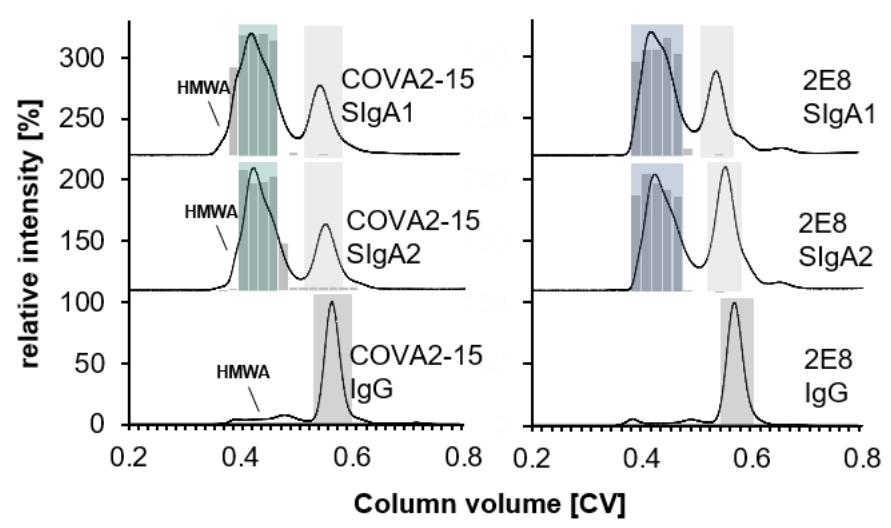

D

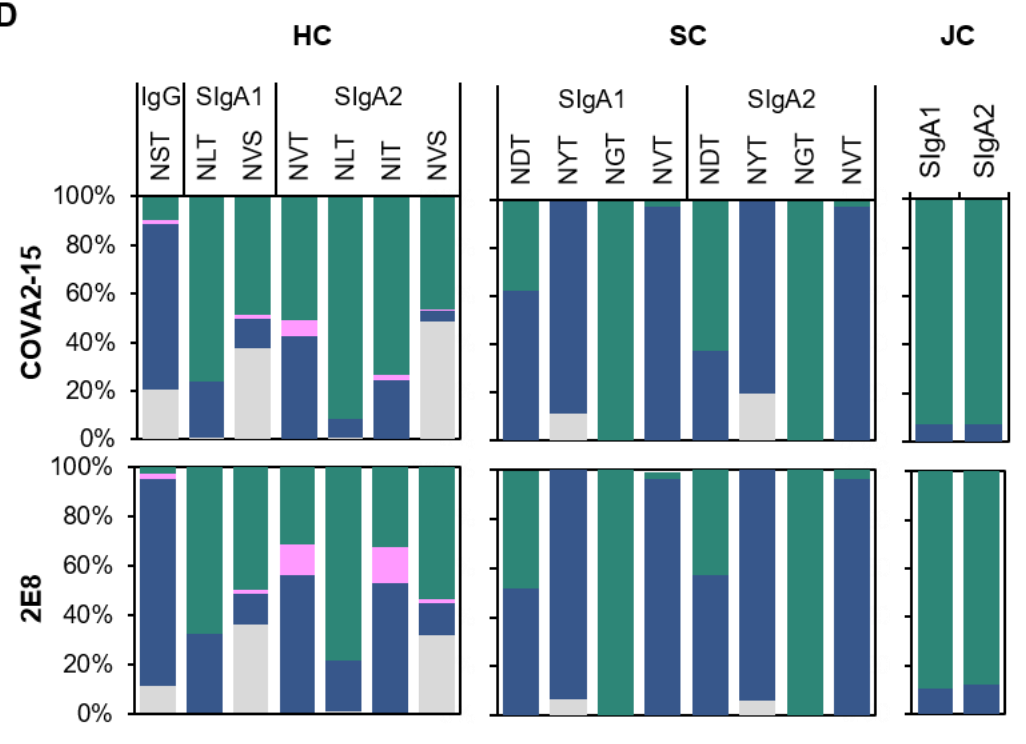

C
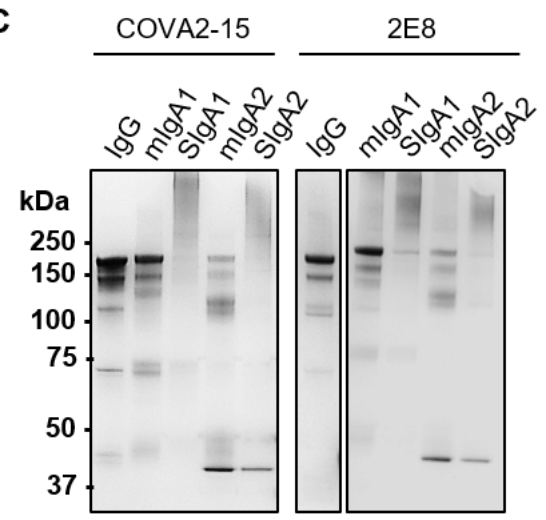

JC

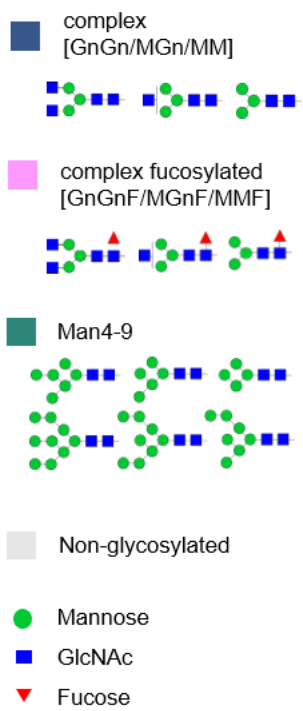

Figure 1: Expression, assembly and purification of monoclonal IgG and different IgA

744 antibodies from $N$. benthamiana plants. (A) $\operatorname{IgG}$, monomeric and secretory $\operatorname{Ig} \mathrm{A} 1$ and $\operatorname{IgA} 2$

745 versions of 4 different mAbs recognizing the SARS-CoV-2 spike proteins were transiently expressed in plants. Expression levels were quantified by sandwich ELISA in crude leaf 
747 extracts. Detection of monomeric IgA and IgG variants was with either HRP-labeled anti-kappa 748 (COVA2-15) or anti-lambda light chain (2E8, COVA1-22, 2-15) antibodies. SIgA antibodies 749 were detected using anti-secretory component antibodies for all SIgA variants. Quantification 750 data represent the mean of two technical repeats of three independent biological repeats \pm SD

751 (B) Normalized size-exclusion chromatograms of affinity-purified IgG, secretory IgA1 and 752 secretory $\operatorname{IgA} 2$ of the COVA2-15 and 2E8 variants from infiltrated $N$. benthamiana $\triangle \mathrm{XF}$ 753 leaves. Values were normalized based on the highest signal of each chromatogram. The ratio 754 of secretory IgA to total IgA in each chromatography fraction was determined by ELISA and 755 the relative amount of SIgA in each fraction is indicated by grey bars. Green, blue and grey 756 boxes indicated pooled fractions. (C) SDS-PAGE under non-reducing conditions of affinity 757 and size-exclusion purified plant-produced IgG, monomeric and secretory IgA1/IgA2 of 758 COVA2-15 and 2E8 visualized by Coomassie Brilliant Blue staining. (D) Site-specific $N$ 759 glycosylation of purified mAbs. Bars represent the relative abundance (\%) of glycoforms 760 present at each glycosite of the heavy chains (HC; IgA1: NLT and NVS, IgA2: NVT, NLT, 761 NIT and NVS, IgG1: NST), the secretory component (SC; NDT, NYT, NGT, NVT) and the 762 Joining chain (JC; NIS). N-glycans are abbreviated according to the ProGlycAn system 763 (www.proglycan.com). The symbols for the monosaccharides are drawn according to the 764 nomenclature from the Consortium for Functional Glycomics. 
Saliva A
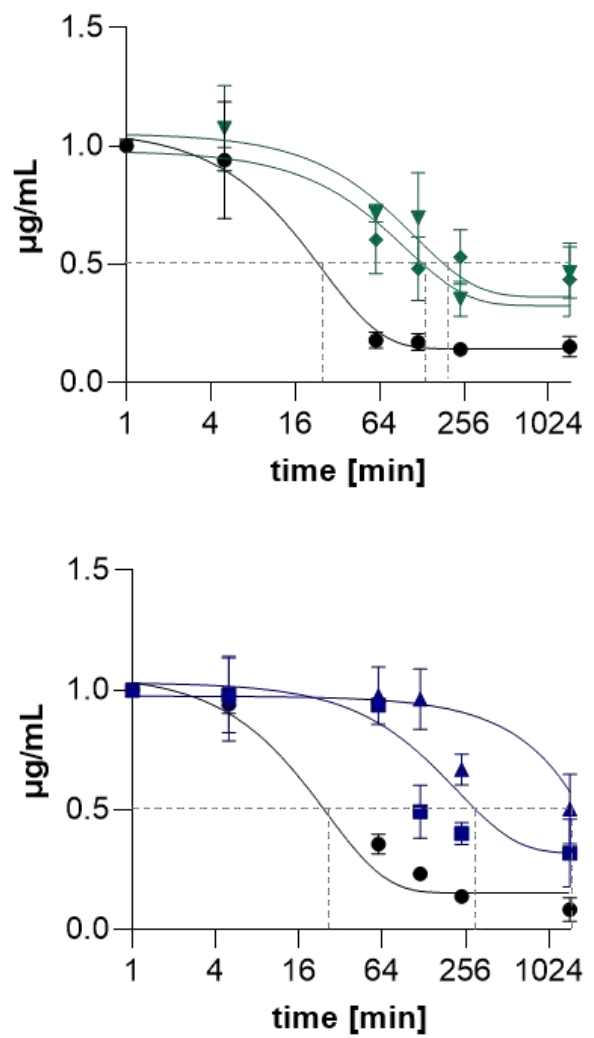

Saliva B

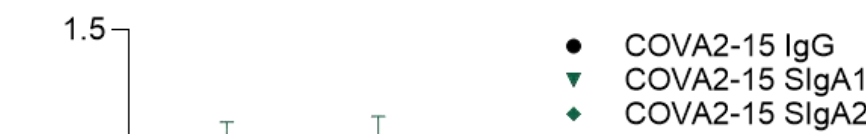

COVA2-15 SlgA1
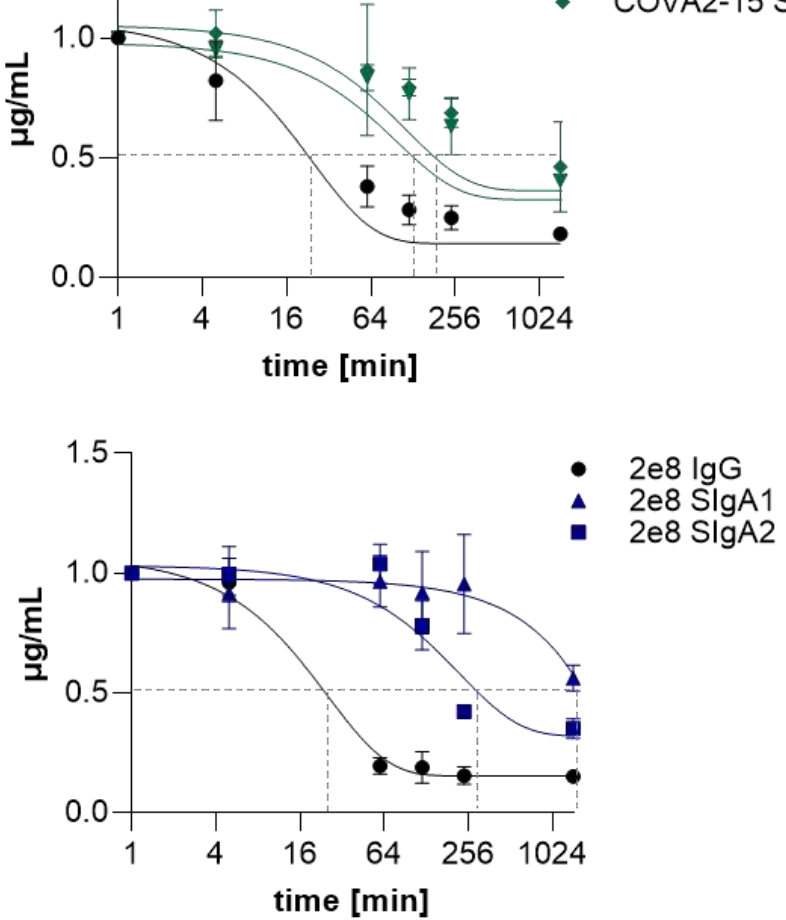

766

767 Figure 2: Stability of COVA2-15 IgG and IgA variants in human saliva. Saliva from two donors (A and B) was mixed with COVA2-15 and 2E8 IgG and SIgA mAb variants and

769 incubated at $37^{\circ} \mathrm{C}$ for the indicated time. Samples were analyzed for binding to RBD and

770 assembly through detection with Fc-specific and SC-specific antibodies. The mean \pm SD of

771 duplicates is shown. Gray dotted lines indicate half-lives of COVA2-15 and 2E8 variants

772 calculated using a one phase decay non-linear regression model (Graphpad Prism). 
A

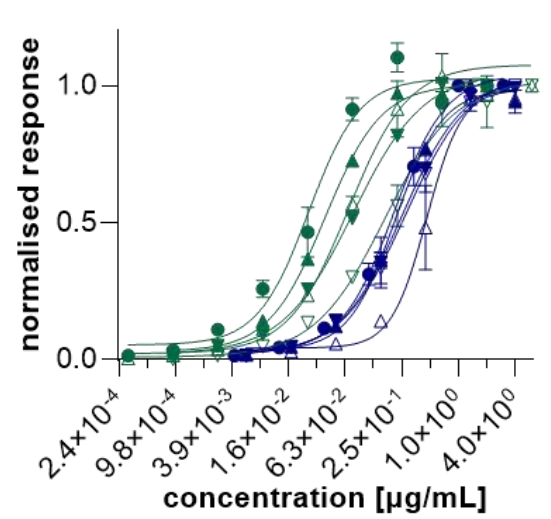

C
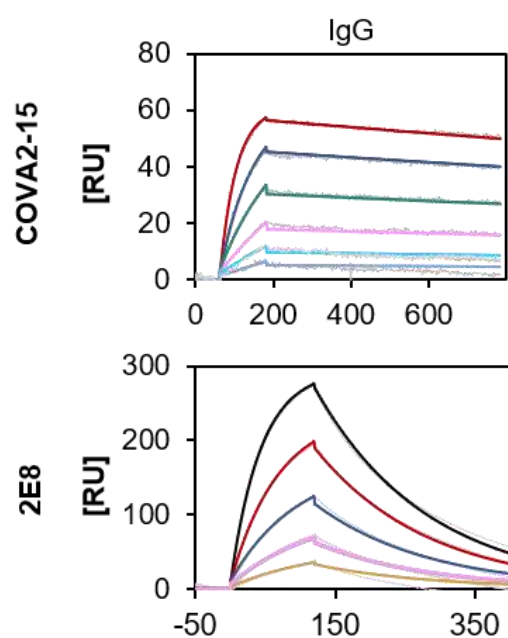

775
B
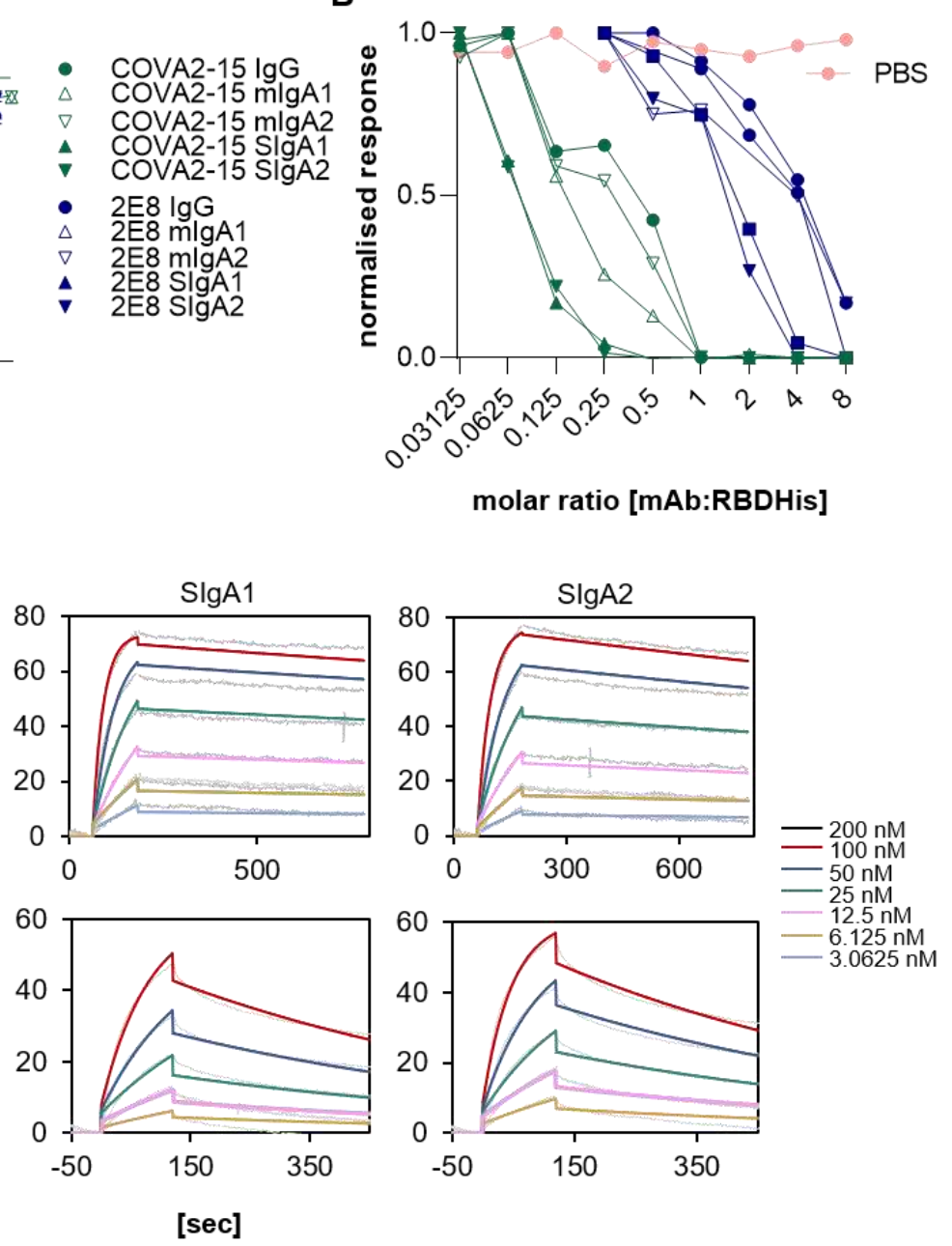

[sec]

Figure 3: Interaction of CoVA2-15 and 2E8 IgG and IgA antibodies with the SARS-CoV-

2 receptor binding domain (RBD). (A) Determination of $\mathrm{EC}_{50}$ values of $\operatorname{IgA}$ and $\operatorname{IgG}$ antiSARS-CoV-2 variants to the receptor binding domain (RBD) by ELISA. Each value is the mean \pm SD from three independent measurements. (B) Inhibition of RBD binding to the ACE2 receptor by COVA2-15 and 2E8 mAb variants was determined by a competitive ELISA assay. Data shown is one representative out of two independent experiments with similar results. (C) Binding kinetics of COVA2-15 and $2 \mathrm{E} 8 \mathrm{mAb}$ variants to RBD were obtained by SPR spectroscopy in multi-cycle kinetic experiments. An anti-His antibody was immobilized on an CM5 chip, RBDHis was captured (50 RU for COVA2-15 IgG, SIgA1 and SIgA2; 100 RU for 2E8 SIgA1, SIgA2; 300 RU for 2E8 IgG), and 5 or 6 different concentrations of the respective 
$786 \mathrm{mAb}$ were injected. The obtained curves were fitted with a 1:1 binding model. Data shown are 787 from one experiment representative of at least two technical repeats. 

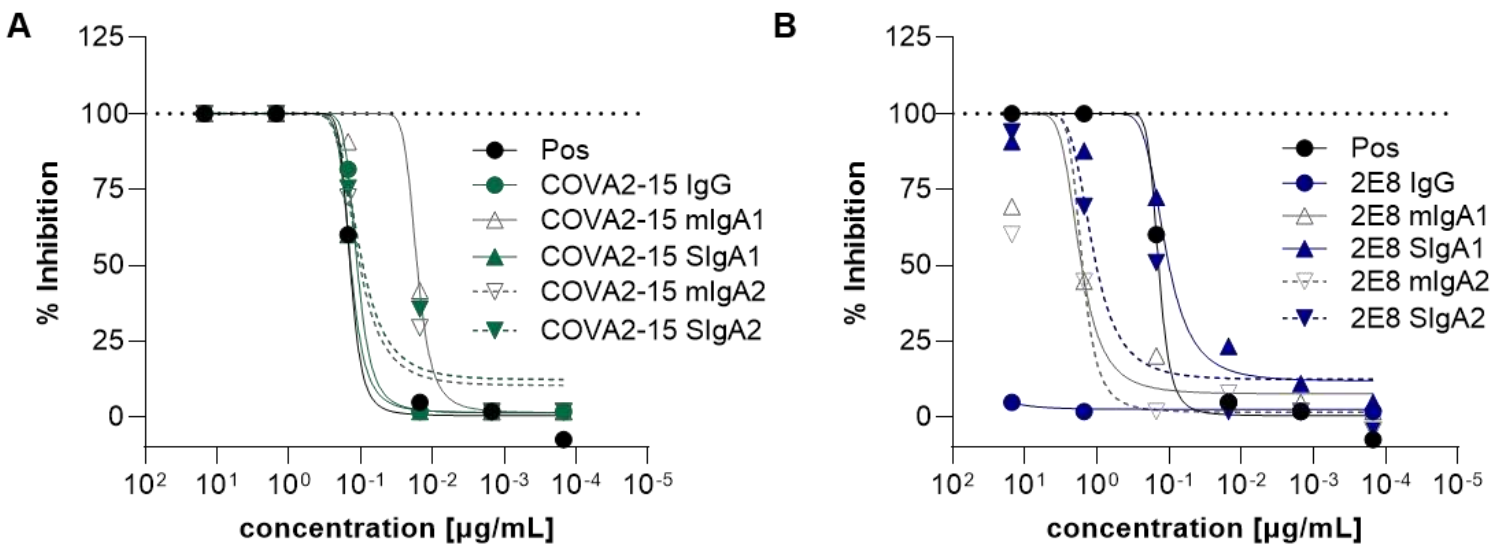

789 Figure 4: Neutralization of SARS-CoV-2 (England 02/2020) by COVA2-15 (A) and 2E8

790 (B) mAbs variants. Neutralization capacity was measured using a PRNT assay on Vero E6

791 cells. MAbs were added in serial 1:10 dilutions starting with $15 \mu \mathrm{g} / \mathrm{mL}$. A positive control

792 (Pos; WHO International Standard of anti-SARS-CoV-2 immunoglobulin, 20/136, NIBSC,

793 UK) was included. The mean of duplicates of one representative out of two experiments with

794 similar results is shown. 
A

\begin{tabular}{cllccc} 
Group & \multicolumn{1}{c}{ Name } & \multicolumn{1}{c}{ Reagent } & (n) & $\begin{array}{c}\text { Antibody } \\
\text { infusion } \\
-24 \mathrm{~h} \text { i.n }\end{array}$ & $\begin{array}{c}\text { SARS-CoV-2 } \\
\text { challenge } \\
0 \mathrm{~h} \mathrm{i.n}\end{array}$ \\
\hline Group 1 & No SARS-COV-2 & Blank & 3 & $\checkmark$ & $\checkmark$ \\
Group 2 & SARS-COV-2 & PBS & 4 & $\checkmark$ & $\checkmark$ \\
Group 3 & IgA Isotype & Human colostrum IgA & 4 & $\checkmark$ & $\checkmark$ \\
Group 4 & IgG Isotype & Human serum IgG & 4 & $\checkmark$ & $\checkmark$ \\
Group 5 & COVA2-15 IgG & COVA2-15 IgG & 6 & $\checkmark$ & $\checkmark$ \\
Group 6 & COVA2-15 SIgA1 & COVA2-15 SIgA1 & 4 & $\checkmark$ & $\checkmark$ \\
Group 7 & COVA2-15 SIgA2 & COVA2-15 SIgA2 & 4 & $\checkmark$ & $\checkmark$ \\
\hline
\end{tabular}

B

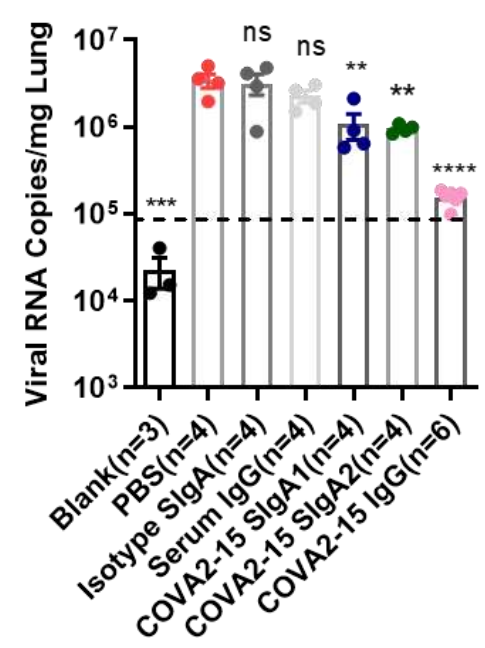

D

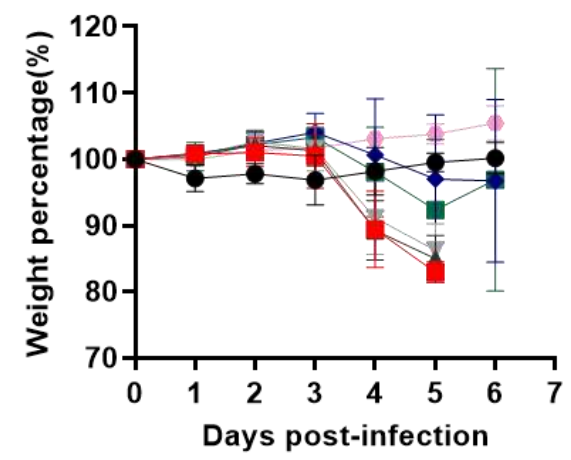

$\longrightarrow \operatorname{Blank}(\mathrm{n}=3) \longrightarrow \operatorname{COVA2}-15 \operatorname{SIgA1}(\mathrm{n}=4)$

- PBS $(n=4)-\operatorname{COVA2}-15 \operatorname{SIgA2}(\mathrm{n}=4)$

$-\quad$ Isotype SIgA $(n=4)-\operatorname{COVA2}-15 \lg (n=6)$

797 Figure 5: Efficacy of intranasally administered COVA2-15 IgG, SIgA1 and SIgA2 in

hACE2 mice. (A) Experimental schedule of COVA2-15 Abs in the prevention and treatment of SARS-CoV-2 infection. The below table summary of groups ( $n=3-6$ mice) with different treatment. (B) Viral loads in lung among 7 groups were measured by qRT-PCR. The name of

801 each group in $X$ axis was indicated according to table in A. Each dot represents one mouse.

802 The limit of detection was $2.3 * 10^{4}$ copies/mg referenced to blank control which was not 
803 infected with SARS-COV-2 (Blank group). Data represent mean \pm SEM. One-way ANOVA 804 was performed to compare treatment group with the PBS control group. ns, no significance; $805 * *, \mathrm{P}<0.01, * * *, \mathrm{P}<0.001 ; * * * *, \mathrm{P}<0.0001$. (C) Survival rate of all 7 groups were recorded 806 and calculated. (D) Body weight of mice among the above 7 groups were recorded. Each line 807 represents data from one group. (E) Representative sections of lung were visualized under the $808 \times 20$ objective. H\&E staining was conducted to analyse the lung inflammation and observed at 809 64-fold magnification. 


\section{Tables}

812 Table 1: Kinetic parameters of COVA2-15 and 2E8 IgG/IgA mAbs to RBD. Rate constants

813 were determined at 5 different concentrations using a 1:1 binding model. Values are shown as

814 mean \pm SD of two technical repeats.

\begin{tabular}{|c|c|c|c|c|c|c|c|c|c|}
\hline & \multicolumn{3}{|c|}{ ka (1/Ms) } & \multicolumn{3}{|c|}{$\operatorname{kd}(1 / \mathrm{s})$} & \multicolumn{3}{|c|}{$K_{\mathrm{D}}(\mathrm{nM})$} \\
\hline COVA2-15 IgG & 332930.6 & \pm & 45451.2 & 0.00024 & \pm & 0.00001 & 0.74 & \pm & 0.08 \\
\hline COVA2-15 SlgA1 & 422888.0 & \pm & 66027.9 & 0.00016 & \pm & 0.00001 & 0.38 & \pm & 0.02 \\
\hline COVA2-15 SlgA2 & 326233.2 & \pm & 91502.2 & 0.00024 & \pm & 0.00003 & 0.76 & \pm & 0.12 \\
\hline 2E8 IgG & 70974.3 & \pm & 12795.4 & 0.00543 & \pm & 0.00080 & 76.93 & \pm & 2.57 \\
\hline 2E8 SIgA1 & 111255.2 & \pm & 137.2 & 0.00147 & \pm & 0.00000 & 13.23 & \pm & 0.02 \\
\hline 2E8 SIgA2 & 155903.3 & \pm & 39703.3 & 0.00155 & \pm & 0.00003 & 10.56 & \pm & 2.79 \\
\hline
\end{tabular}

815 


\section{Supplementary Files}

This is a list of supplementary files associated with this preprint. Click to download.

- Supplementarylnformation211105.pdf 